Research Paper

\title{
Mechanical and structural characterization of tibial prosthetic interfaces before and after aging under simulated service conditions
}

\author{
A. Cavaco ${ }^{a, b}$, A. Ramalhoc ${ }^{c}$ S. Pais ${ }^{b}$, L. Durães ${ }^{a, *}$ \\ ${ }^{a}$ CIEPQPF, Department of Chemical Engineering, University of Coimbra, Rua Silvio Lima, 3030-790 Coimbra, Portugal \\ ${ }^{b}$ ESSUAlg, School of Health, University of Algarve, Av $v^{a}$. Dr. Adelino da Palma Carlos, 8000-510 Faro, Portugal \\ ${ }^{c}$ CEMUC, Department of Mechanical Engineering, University of Coimbra, Rua Luís Reis Santos, 3030-788 Coimbra, \\ Portugal
}

\section{A R T I C L E I N F O}

\section{Article history:}

Received 19 September 2014

Accepted 5 December 2014

Available online 18 December 2014

Keywords:

Tibial prosthesis

Elastomeric interface materials

Aging in sweat

Dynamic-mechanical

characterization

\begin{abstract}
A B S T R A C T
Prosthesis interface is one of the most important components to promote individual's health and comfort, as it establishes direct contact with the skin and transfers loads generated during gait. The aim of this study was to mechanically characterize, three commercial interfaces (block copolymer, silicone gel and silicone elestomer), under static and dynamic conditions, before and after undergoing a process of chemical aging in synthetic sweat for periods up to 90 days. Static mechanical compression tests were performed on the materials, as well as fatigue tests to assess their static and dynamic mechanical behaviors, respectively. For the second, a sinusoidal load was applied with an appropriate range of deformation for each material. Several analytical techniques were also used to characterize the materials, namely Fourier Transform Infrared Spectroscopy (FTIR), X-ray diffraction (XRD), and morphology characterization by Scanning Electron Microscopy (SEM). All the tested materials have strong viscoelastic behavior, showing a linear response for small deformations, followed by a nonlinear behavior for higher deformation. The block copolymer and the silicone gel are affected by aging in synthetic sweat in a similar way, with a significant increase of their rigidity after 30 days, followed by a progressive reduction. The silicone elastomer displays a continuous increase of rigidity along the 90 days of storage, being the most sensitive to aging affects. It also exhibits the lowest stiffness value, being suitable for uses that require maximum comfort. All materials demonstrate chemical and structural stability under service simulated conditions.
\end{abstract}

(c) 2014 Elsevier Ltd. All rights reserved.

\footnotetext{
*Corresponding author. Tel.: +351 239798737; fax: +351 239798703.

E-mail address: luisa@eq.uc.pt (L. Durães).
} 


\section{Introduction}

Amputation causes profound changes in individual's health, affecting their perception of comfort (Carvalho, 2003; Martín and Pérez, 2009; Ramos et al., 2007), quality of life (De Godoy et al., 2004), activity level and society participation (Huang et al., 2001; Sinha et al., 2011). In this context, the correct selection and application of the prosthesis determines the success of the rehabilitation process (Klute et al., 2001; Linde et al., 2004). The prosthesis must fulfill certain conditions, such as comfort, donning, weightlessness, durability, good mechanical function, pleasing esthetics, easy maintenance and low cost (Huang et al., 2001).

The proper application of the prosthesis is directly dependent on the correct selection of materials, according to their structure, properties and behavior (Lunsford and Contoyannis, 2009). Of all prosthesis components, the interface is specially important, due to its function in establishing a direct connection between the stump (i.e. the individual) and the prosthesis, being responsible for the transmission of ground reaction forces, damping gait loads and tissue protection (Huang et al., 2001; Klute et al., 2001). Silicone-based materials and polymeric elastomers, for instance urethane elastomers, have been used in its confection. The inadequacy of the interface may result in discomfort and serious skin problems, such as ulcers, contact dermatitis, eczema, epidermoid cysts and fungal infections or bacterial infections (Levy, 1980; Mak et al., 2001; Meulenbelt et al., 2009).

In the absence of joints and tissues for cushioning the impact on the amputated limb, the stump becomes vulnerable to the transmission of loads (Berge et al., 2005; Klute et al., 2001). The repetitive impact load that exists between the heel and the ground when walking, coupled to the referred absence of anatomical structures, can lead to pain on the stump (Klute et al., 2001).

To better understand the mechanical behavior of interfaces, exposed to these load cycles, Sanders et al. (2000) investigated the compressive stiffness of eight types of materials: spenco, poron, silicone, soft pelite, medium pelite, firm plastozote, normal plastozote and nickelplas. Spenco, poron and silicone were recommended for situations where it is desirable to keep the interface thickness and volume, as these materials have high capacity to recoverable deformation. The nickleplast behaved with linearity but with greater stiffness, suggesting that it does not offer a significant effect of cushioning. In a later stage, more fifteen commercial interfaces were tested under compression, tension and shear forces by Sanders et al. (2004), including specimens made of silicone elastomer, silicone gel and polyurethane. The silicone gel was softer during the compression tests and more like the biological tissues of the stump, suggesting that it would be more appropriate to cushion and protect the bony prominences of the stump. The silicone elastomer has shown more compression rigidity, as well as the polyurethane sample, proving to be advantageous for stumps with excess soft tissue, as they do not add more strain to the already existing soft tissue. The results of the shear tests led to the conclusion that the silicone elastomers and polyurethane are the most suitable materials for stumps with high proportion of soft tissue, because they prevent the slide of the stump in the socket, while silicone gels are appropriate for stumps with abundant bony prominences, due to the already mentioned cushioning effect (Klute et al., 2010; Sanders et al., 2004).

Moreover, Selinger (2004) identified only three published studies comparing the properties of various materials used in interfaces. These studies report that the physical properties of the silicone gel and silicone elastomers are quite similar. They indicate that elastomers are often preferred due to their stiffness properties and compressive strength, while polyurethane shows higher coefficient of friction.

Dynamic mechanical tests were conducted on interface materials by Emrich and Slater (1998), who investigated samples of bock-lite, pedilin, silicone and polyurethane. The interfaces were tested under cyclic compressive load, cyclic abrasive cutting load and friction. Bock-lite and silicone needed the highest number of cycles to failure under mechanical compressive loads, in contrast to polyurethane and pedilin that needed a lower number of cycles. It was also possible to verify that the sample of bock-lite resisted 15 times more cycles than pedilin under abrasion shear forces.

The relationship between normal and shear components of the force applied by the socket in the stump is very complex. It changes along the residual limb surface and depends on the loads exerted during gait. It also depends on the design of prosthetic units outside the socket. Exact evaluation of pressures requires the ability to deal with the viscous-elastic properties of prosthetic interfaces, residual limb skin, and soft tissues compressed against the bones (Pitkin, 2010).

In summary, the soft interface plays a key role in gait performance and comfort because it acts as a coupling body between the limb and the hard socket, having the following goals, from the mechanical point of view: transmit the load; distribute the pressure by the limb surface, reducing interface stresses; smooth the peak of the transmitted forces; adapt the motion between the limb and the interface by deformation, preventing the relative motion in order to minimize the occurrence of pressure ulcers. The availability of rigidity and damping characteristics of the interfaces materials is thus crucial to allow the use of numerical models to estimate the values of the contact forces and the corresponding stress distribution on the contact limb-prosthesis. Only assessing the material properties will be possible to optimize the interface material selection and adapt its geometry to the needs of the amputee, essentially concerning his activity. For this purpose, a functionality classification method was developed by DMERC Medicare Advisory (1994). This scale has five levels, from K0 (highly dependent - patients do not have the ability or potential to ambulate or perform transfers safely with or without assistance; in this case the prosthesis does not enhance their quality of life or mobility) to K4 (highly independent - patients have skills or potential to ambulate with a prosthesis and exceeds daily living basic skills activities, namely those involving high levels of impact, stress or energy consumption during activity). This classification method is normally used by manufacturers to classify the suitability of prosthesis components according to patients functional level (Berke, 2007). 
Although several studies have been conducted for the mechanical characterization of soft interfaces materials (Berge et al., 2005; Emrich and Slater, 1998; Sanders et al., 1998, 2000, 2004), it is difficult to compare the obtained results because the test conditions were not similar, especially concerning the strain rate. Moreover, the environmental test conditions do not reproduce the service conditions of prosthesis interfaces.

For other biomedical applications, namely for soft interface dentist materials, the effect of aging in different chemical environment were investigated (Leite et al., 2010). The experience and opinion of tibial amputees show that the interfaces lose the comfort in a short period of time. Considering comfort as a fundamental property in their quality of life (van de Weg and van de Windt, 2005), we assume these comfort lose is induced by the change of mechanical properties, caused not only by the constant load cycles as well as by the existing thermal-chemical environment within the interface.

Therefore, the aim of this study was to mechanically characterize, under static and dynamic loading conditions, three commercial interface materials. In addition, each material was characterized in post-processed conditions after different aging periods in a synthetic sweat chemical solution. To our knowledge, these aging tests and subsequent characterization were never conducted in the studied interface materials.

\section{Materials and methods}

\subsection{Materials}

The materials in study, I, II and III, are representative of the most used materials in interfaces for tibial prosthesis. The selected materials were:

Material I - AKDT Locking, Extreme Gel for High activity, block copolymer;

Material II - Clear liner basic, Streifeneder 1S3 silicone gel; Material III - First class liner, Streifeneder 1S5 silicone elastomer.

These interfaces usually have a variable thickness to provide better accommodation of the stump, being thicker in the distal region and thinner in the proximal region. The samples for the different tests were extracted directly from entire new interfaces (fresh/original interfaces), therefore, and because they are different materials, they exhibit some variability in their thickness, albeit this was kept as minimum as possible, and all tests were adjusted for these differences.

\subsection{Aging process}

In order to simulate natural material aging, resulting from the interface use in contact with body sweat, eight pieces from each material $(50 \mathrm{~mm} \times 50 \mathrm{~mm}$ ) were kept soaked in a synthetic sweat bath, duly sealed in a glass container with a controlled temperature of $25^{\circ} \mathrm{C}$. The synthetic human sweat was prepared according to the ISO 3160-2 standard ( $\mathrm{NaCl}-20 \mathrm{~g} / \mathrm{L}, \mathrm{NH}_{4} \mathrm{Cl}-17.5 \mathrm{~g} / \mathrm{L}$, lactic acid $-15 \mathrm{~g} / \mathrm{L}$, acetic acid $-5 \mathrm{~g} / \mathrm{L}$ ) with a $\mathrm{pH}$ of 4.7 . Two pieces of each material were removed for characterization after aging in the sweat bath for 30, 60 and 90 days.

\subsection{Mechanical characterization - static loading}

The mechanical experiments under static loading were conducted in a Shimadzu AG-X plus universal testing machine, adjusted to a compression test. This machine has a load cell of $5 \mathrm{kN}$ and tests were done with a loading rate of $5 \mathrm{~mm} / \mathrm{min}$ and a data acquisition rate of $10 \mathrm{~Hz}$. During tests, there was no radial constriction of specimens. All materials, in fresh condition and after being aged in several steps, were compressed with a $16 \mathrm{~mm}$ diameter punch until reaching a strain of 0.4. The active area of compression was the area of the circular punch. For each sample, 5 replicas were done.

\subsection{Mechanical characterization - dynamic loading}

An Instron ElectroPuls ${ }^{\mathrm{TM}}$ fatigue machine was used to perform the dynamic compression tests. In these tests, all the samples were tested applying a cyclic sinusoidal compression with a frequency of $10 \mathrm{~Hz}$ and $0.3 \mathrm{~mm}$ of displacement peak-to-peak amplitude, except for material II that was tested with amplitude of $0.1 \mathrm{~mm}$. The materials were previously prepared in discs with $18 \mathrm{~mm}$ of diameter and were compressed between flat holders without radial restriction. Also in this case, the materials were tested in the fresh state and after aging.

\subsection{Chemical structure of the interface materials}

Fourier Transform Infrared Spectroscopy (FTIR) analysis was used to assess the chemical structure of the three interface materials studied in this work, in the as-received condition and after aged during three months in synthetic sweat. The analysis was carried out in a Jasco V550 FTIR spectrometer, using the ATR (Attenuated Total Reflectance) module. The spectra were collected in the wavenumber range between 500 and $4000 \mathrm{~cm}^{-1}$. The vibration bands found in each spectrum were ascribed to the chemical bonds/groups present in the corresponding samples, considering the characteristic vibration frequencies of organic and silica based materials published in the literature (Al-Oweini and El-Rassy, 2009; Becker et al., 1997).

\subsection{Structural and morphological analysis}

Samples were also analyzed on a Philips diffractometer, model X 'Pert, in order to follow the evolution of the microstructure along the aging period. The analyses were performed in $\theta-2 \theta$ geometry (parallel beam). The used accelerating voltage was $40 \mathrm{kV}$ and the current intensity was $35 \mathrm{~mA}$. The used anticathode was cobalt, with a $\mathrm{K} \alpha 1$ and $\mathrm{K} \alpha 2$ wavelengths of 0.178896 and $0.179285 \mathrm{~nm}$, respectively. The angles range was between $5^{\circ}$ and $100^{\circ}(2 \theta)$, with a step size of $0.025^{\circ}$ and an acquisition time of $1 \mathrm{~s} / \mathrm{step}$.

The morphology of the different interface materials was observed, on fractured surfaces, by a Scanning Electron Microscope (SEM) Philips XL30 TMP. All of the tested specimens were sputter-coated with gold in order to allow a better observation. The images were obtained either with secondary or backscattered electrons to improve the contrast. 


\section{Theoretical substantiation and data treatment procedure}

\subsection{Constitutive models for low strain rate tests}

Results of all static tests were analyzed using nominal stressstrain values; the compressive stress $(\sigma)$ was assumed by the quotient between the measured force and the active area of the sample, and the strain $(\varepsilon)$ was calculated as the measured displacement divided by the thickness of the material, assessed on unloaded condition.

These materials behave with a linear response up to $\varepsilon_{\text {elas }}$ (limit of linear strain) and with a nonlinear behavior for higher deformation. Therefore, a constitutive law with two branches is required to correlate the stress and the strain. Eq. (1) presents a double branch law with a linear part up to $\varepsilon_{\text {elas }}$ and a more complex equation corresponding to the nonlinear portion.

$\sigma=\left\{\begin{array}{c}A \varepsilon, \varepsilon<\varepsilon_{\text {elas }} \\ A \varepsilon+B\left(\varepsilon-\varepsilon_{\text {elas }}\right)^{n}, \quad \varepsilon \geq \varepsilon_{\text {elas }}\end{array}\right.$

For the elastic domain one parameter is enough to characterize the stress and strain behavior, while for the nonlinear domain three more parameters are required to fit the stressstrain evolution. The A linear parameter is defined by the value of the elastic modulus (or Young's modulus) found by fitting a linear equation to the first part of the curve. For the second branch of the Eq. (1), a power term was used because it fits the data better than other alternatives and is a common constitutive law applied to this type of materials (Sanders et al., 2004). Thus, parameter $B$ is a constant that multiplies by the non-elastic portion of the deformation, raised to a power constant, $n$.

\subsection{Constitutive models for dynamic mechanical tests, viscoelasticity and energy dissipation}

Viscoelastic materials exhibit both viscous and elastic characteristics when undergoing deformation.

In a first approach, the Kelvin-Voigt model was used to describe the response of the tested materials under dynamic loading. This model includes a spring in parallel with a dashpot (Fig. 1a). This is a suitable configuration to describe viscoelastic materials, which exhibit time-dependent strain revealing the elastic and the viscous effects, as expressed by Eq. (2). The elastic component corresponds to the first linear term of the equation, while the viscous component is a function of strain rate and corresponds to the second term of Eq. (2).

In this equation $\sigma(t)$ is the stress, $\eta$ is the dynamic viscosity of the material and $d \varepsilon / d t$ is the strain rate.

$\sigma=E \varepsilon(\mathrm{t})+\eta \frac{\mathrm{d} \varepsilon(\mathrm{t})}{\mathrm{dt}}$

However, the experimental results revealed that the materials under study behave differently from the KelvinVoigt model. These materials under compression are well fitted by a spring in parallel with a dashpot, but when the stress is relieved only a linear behavior is observed. Thus, the damper only works when there is a compression solicitation $(\dot{x}<0)$. To explain that behavior, a modified Kelvin-Voigt model was proposed as displayed in Fig. 1b, which corresponds to Eq. (3). The unidirectional valve in parallel with the dashpot means that the dashpot only works during the compression half-period. Therefore, the dynamic behavior can be described by a linear differential equation, which is the main advantage of the proposed solution.

$\sigma=\left\{\begin{array}{c}E \varepsilon(\mathrm{t})+\eta \frac{\mathrm{d} \varepsilon(\mathrm{t})}{\mathrm{dt}}, \quad \dot{\mathrm{x}}<0 \\ E \varepsilon(\mathrm{t}), \mid \dot{\mathrm{x}} \geq 0\end{array}\right.$

Each viscoelastic material was studied by analyzing the dynamical response to a sinusoidal load, assuming an appropriate range of deformation for each material. Stress-strain graphics of each loading cycle display a semielliptical loop shape (Fig. 1C), which agrees with the constitutive proposed model. Through these curves it is possible to know the quantity of the specific energy dissipated for each excitation cycle, which is proportional to the area of the loop.

\subsection{Data treatment procedure}

\subsubsection{Static tests}

The experimental data obtained from static tests were treated using a suitable data analysis procedure to calculate the parameters of Eq. (1), namely A, B and $n$, that better correlate the data points.

First, a linear regression was used to find the limit of linear strain, $\varepsilon_{\text {elas}}$, and the elastic modulus, A. The limit of linear strain $\left(\varepsilon_{\text {elas }}\right)$ was calculated by an optimization procedure, adding points to the linear fitting of the experimental stress versus strain until reaching the maximum correlation factor.

To define the nonlinear stage, it was necessary to introduce a variable transformation on Eq. (1), as indicated by Eq. (4).

$\sigma^{\prime}=B \varepsilon^{\prime n},\left\{\begin{array}{c}\sigma^{\prime}=\sigma-A \varepsilon \\ \varepsilon^{\prime}=\varepsilon-\varepsilon_{\text {elas }}\end{array}\right.$

The least squares method was used to adjust the power function, and the solution that better fitted the data points allowed to define the nonlinear phase, establishing the best values of $B$ and $n$ for Eq. (4).

The accuracy of the developed models was assessed by calculating the mean squared error normalized by the rms value of the stress along the loading phase.

\subsubsection{Dynamic tests - sinusoidal wave excitation}

Assuming the modified Kelvin-Voight model previously presented, the force transmitted by the viscoelastic material can be calculated using the stress values of Eq. (3), being $E$ and $\eta$ the parameters of the material constitutive model. The procedure used to adjust the best values of $E$ and $\eta$ is based on a two-step fitting, as follows:

1 From the semielliptical loop of $\sigma-\varepsilon$, Fig. 1c, the linear portion is used to fit a straight line being the elastic modulus $\mathrm{E}$ the corresponding slope;

2 The viscosity $\eta$ is optimized by a least squares method, minimizing the error between the stress calculated by the Eq. (3) in comparison to the experimental stress acquired during one cycle of the steady-state regime. 

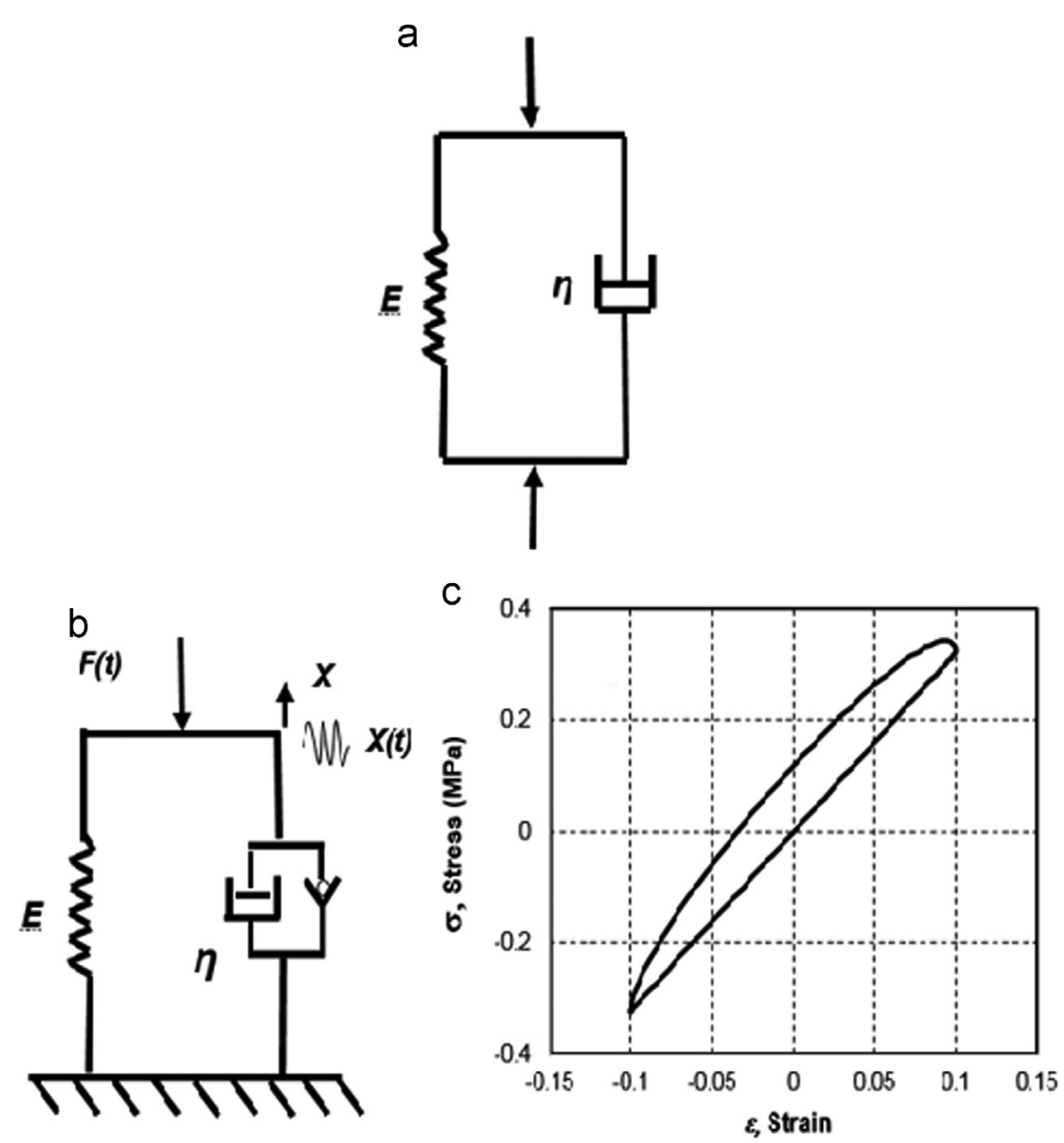

Fig. 1 - (a) Kelvin-Voigt model, (b) constitutive modified Kelvin-Voight model and (c) the corresponding stress-strain hysteresis loop (modified model).

The energy dissipated in each cycle, is another parameter that allows to compare the theoretical model and the experimental results and also to quantify the ability of each material to dissipate energy. The area of the semielliptical shape of stress versus strain in dynamic loading represents the energy dissipated per volume unit of material during one stress cycle, $E_{D}$, Eq. (5).

$E_{D}=\oint_{\varepsilon} \sigma \mathrm{d} \varepsilon$

\section{Results and discussion}

\subsection{Static mechanical tests}

\subsubsection{Behavior of the non-aged materials}

All the materials under study were firstly tested in the asreceived condition, with no degradation or aging. Fig. 2 displays the stress - strain evolution for the three materials tested on the fresh condition. The considered limit of strain was 0.4 because, for higher deformation, the behavior of material I was significantly affected by the compression holders. As expected, the static results showed a first linear phase, with constant stiffness, followed by a continuous increasing of the stiffness.
Material I displays the lowest stiffness when compared to the other two materials, showing a higher strain whatever the stress considered.

Material III shows an elastic modulus comparable to material I. However, above the elastic limit, it exhibits a more pronounced rise of the stiffness with the increase of the strain. Material II has the highest elastic modulus and behave almost linearly for the entire strain domain considered.

Table 1 presents the results of the parameters obtained by fitting the model of Eq. (1) to data of the static mechanical tests.

Sanders et al. (2004) and Covey et al. (2000) tested similar materials to materials II and III, the silicone gel and silicone elastomer, respectively. Although the materials similarity, the results were slightly different. In our study, materials II and III present higher values of the elastic modulus (constant A) and lower limit of linear strain ( $\varepsilon_{\text {elas }}$ ); this should be due to the use of different testing procedures, for instance the loading rate, and mainly because the properties of these kind of materials varies significantly with the supplier due to small variations of the preparation routines.

Fig. 2 displays a comparison between the theoretical model based on the constitutive materials law and the experimental data. The accuracy/agreement of the theoretical model when compared to the compression behavior of the materials under 


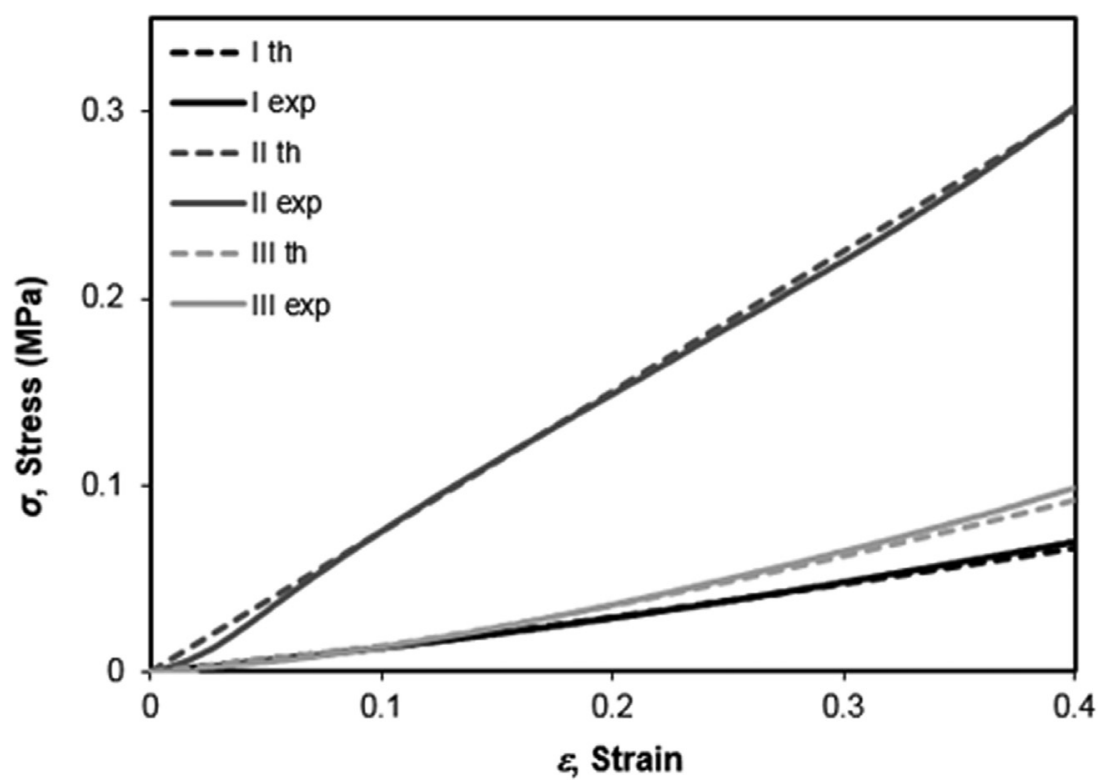

Fig. 2 - Comparison of the experimental stress-strain curves with the results of the theoretical model for the materials in fresh condition.

Table 1 - Constants of the characteristic stress-strain curves of the interface materials in fresh condition and after aging in synthetic sweat, from 0 to 90 days.

\begin{tabular}{|c|c|c|c|c|}
\hline \multirow[t]{2}{*}{ Days of aging } & $\mathrm{A}(\mathrm{MPa})$ & B (MPa) & $\varepsilon_{\text {elas }}$ & $n$ \\
\hline & \multicolumn{4}{|l|}{ Material I } \\
\hline 0 (fresh state) & $0.133 / 100 \%$ & $0.072 / 100 \%$ & $0.109 / 100 \%$ & $1.36 / 100 \%$ \\
\hline 30 & $0.189 / 142 \%$ & $0.158 / 219 \%$ & $0.081 / 74 \%$ & $1.45 / 107 \%$ \\
\hline 60 & $0.147 / 110 \%$ & $0.249 / 346 \%$ & $0.074 / 68 \%$ & $1.87 / 138 \%$ \\
\hline \multirow[t]{2}{*}{90} & $0.112 / 84 \%$ & $0.105 / 146 \%$ & $0.067 / 61 \%$ & $1.67 / 123 \%$ \\
\hline & Material II & & & \\
\hline 0 (fresh state) & $0.753 / 100 \%$ & - & - & - \\
\hline 30 & $1.187 / 158 \%$ & - & - & - \\
\hline 60 & $0.862 / 114 \%$ & - & - & - \\
\hline \multirow[t]{2}{*}{90} & $0.812 / 108 \%$ & - & - & - \\
\hline & Material III & & & \\
\hline 0 (fresh state) & $0.173 / 100 \%$ & $0.208 / 100 \%$ & $0.087 / 100 \%$ & $1.46 / 100 \%$ \\
\hline 30 & $0.236 / 136 \%$ & $0.465 / 224 \%$ & $0.071 / 82 \%$ & $1.77 / 121 \%$ \\
\hline 60 & $0.209 / 121 \%$ & $0.533 / 256 \%$ & $0.044 / 51 \%$ & $1.73 / 118 \%$ \\
\hline 90 & $0.275 / 159 \%$ & $0.758 / 364 \%$ & $0.040 / 46 \%$ & $1.87 / 128 \%$ \\
\hline
\end{tabular}

the static mechanical test is evident in this figure. The obtained errors being lower than $5 \%$.

\subsubsection{Aging effect}

There is a recognized inherent deterioration of the mechanical properties of the interface materials with their use over time. With the simulation of aging in synthetic sweat performed in this work, it is possible to understand the behavior of these materials through time under service conditions.

Fig. 3 shows the stress-strain behavior of material I after the different periods of aging and the corresponding evolution of this material's characteristics with the aging time can be consulted in Table 1. The elastic modulus increases over than $40 \%$ for 30 days of aging and after it starts decreasing, reaching a minimum of $84 \%$ of the initial value induced by 90 days of storage in synthetic sweat. Both the limit of linear strain and the power exponent display a monotonic variation, the first decreasing with the aging time, while the $n$ value increases.

Fig. 4 and data in Table 1 show the effect of the aging in synthetic sweat solution on the mechanical behavior of material II. Concerning the Young's modulus (constant A), material II behaves in a similar way to material I. It presents an increase in the first stage of aging, reaching the maximum increment of $58 \%$ in relation to the original value after 30 days of aging in synthetic sweat. By continuing the aging, the 


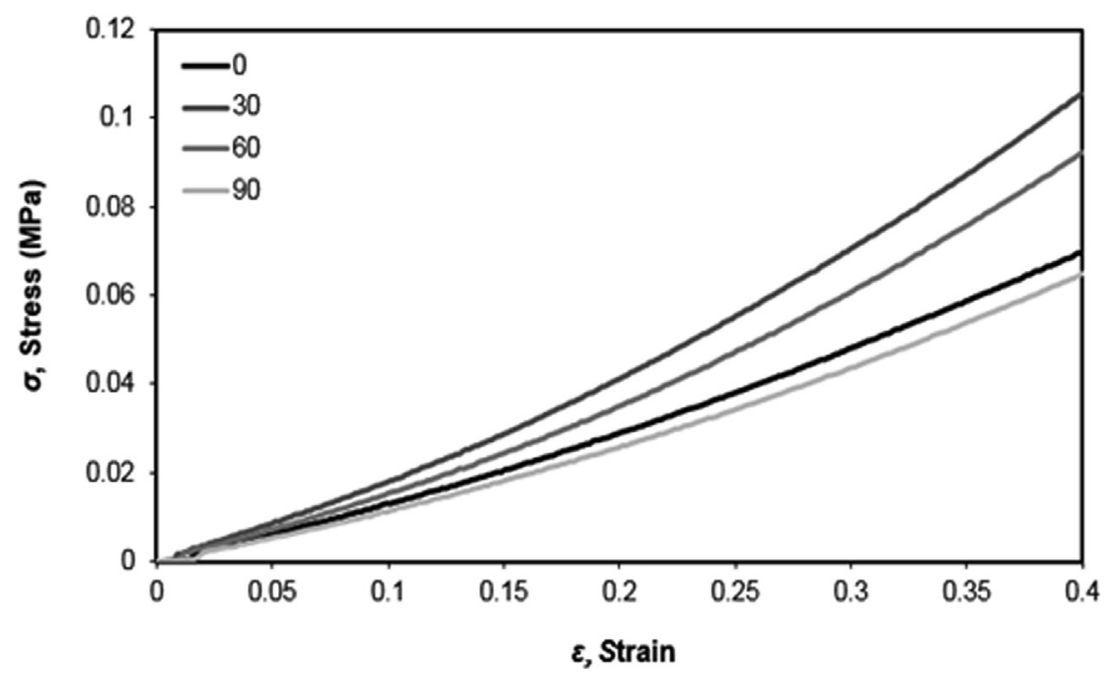

Fig. 3 - Effect of the aging period in synthetic sweat, from 0 to 90 days, on the static mechanical behavior of material I.

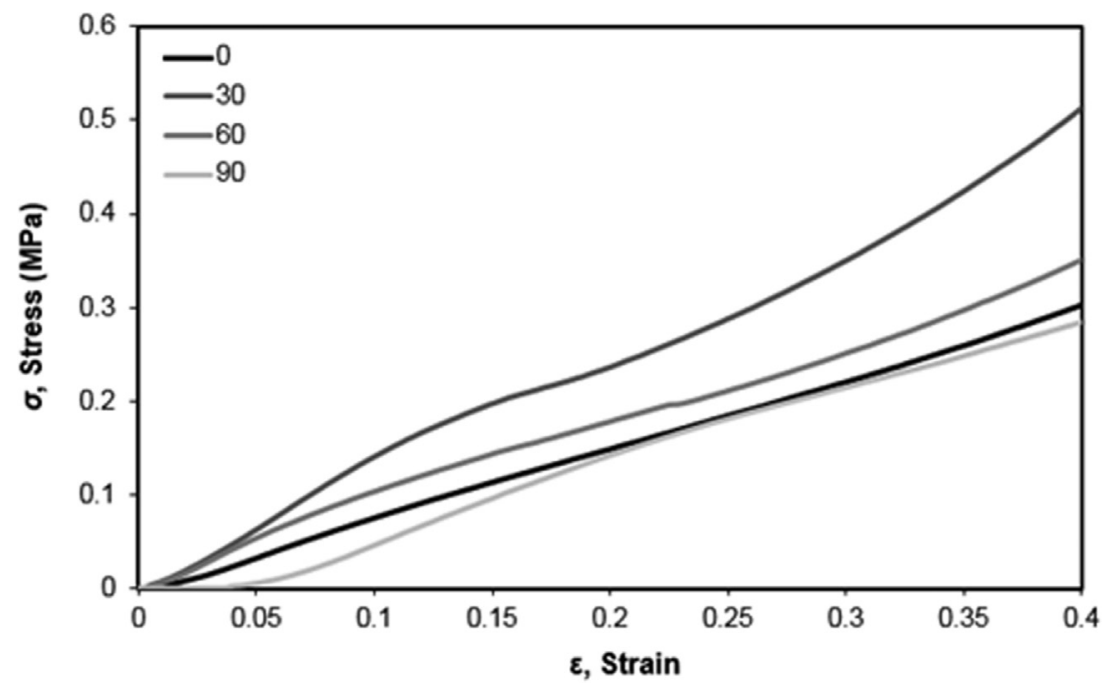

Fig. 4 - Effect of the aging period in synthetic sweat, from 0 to 90 days, on the static mechanical behavior of material II.

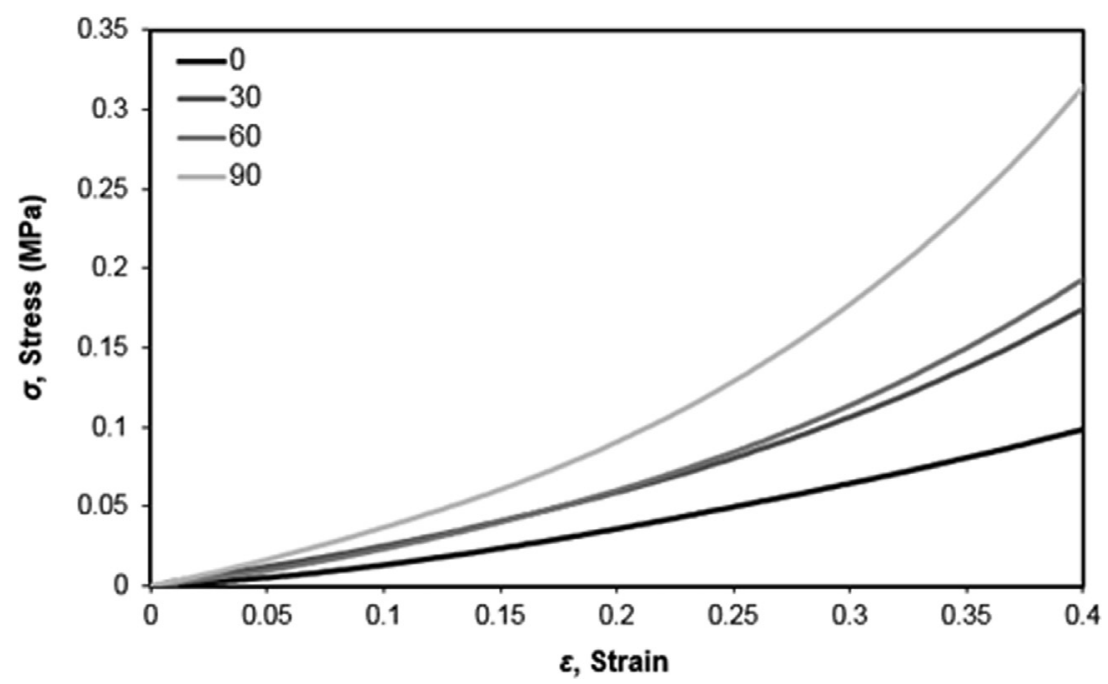

Fig. 5 - Effect of the aging period in synthetic sweat, from 0 to 90 days, on the static mechanical behavior of material III. 
Young's modulus decreases and reaches almost the original value after 90 days of aging.

Through Fig. 5 and analyzing the parameters in Table 1, it is evident that material III behaves in a different manner when compared to the other tested materials. The Young's modulus and all the other characteristic constants, except the limit of linear strain, display a continuous increase with the aging time, reaching their maximum values after 90 days of aging.

\subsection{Dynamic mechanical tests}

\subsubsection{Behavior of the non-aged materials}

The stress-strain curve in dynamic loading shows a loop that is called hysteresis loop. The area of the loop represents the energy lost during one loading cycle. After few cycles, the hysteresis loop reaches a steady shape and, in Fig. 6, it is possible to observe the typical loop that was assessed from each material in the fresh condition. The three studied soft interface materials display marked differences concerning both rigidity and damping. Material II is significantly more rigid than the other two, as was also observed in the static tests. Material III is the one that dissipate more energy while material I behave almost like a linear elastic material with a little dissipation.

Comparing the results related to the fresh state of the materials presented in Table 2 with those of Table 1 , it is possible to conclude that all materials exhibit strong viscoelastic behavior. In fact, for the non-aged samples, comparing the Young's modulus shown in Table 1 (constant A) with the corresponding values of the bulk modulus (column $E^{\prime}$ ) in Table 2, it is possible to see an increase of about one order of magnitude for the dynamic results. The viscous behavior can also be confirmed by the high values of $\tan \delta$.

Fig. 6 compares the experimental behavior of the three tested materials with the estimated values obtained from Eq. (3), using in the later the modified Kelvin-Voight model. The energy dissipated by each cycle was used to evaluate the accuracy of the models and the differences are lower than $3 \%$.

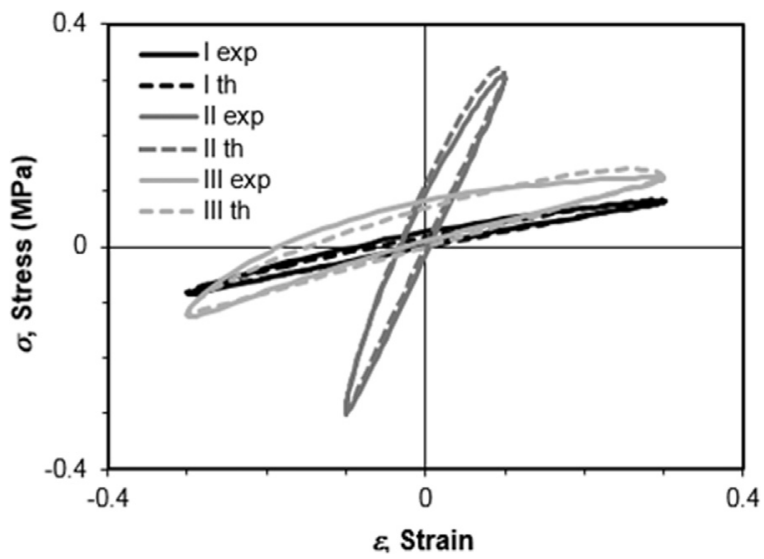

Fig. 6 - Comparison of the experimental stress-strain dynamic loops with the results of the theoretical model for the materials without aging.
Table 2 - Bulk modulus, E', lost modulus, E', tangent of the phase angle, $\tan \delta$, and energy dissipated per volume unit, $E_{\mathrm{D}}$, for the interface materials in the fresh condition and after aging in synthetic sweat, from 0 to 90 days.

\begin{tabular}{lclll}
\hline \multirow{2}{*}{ Days of aging } & $E^{\prime}(\mathrm{MPa})$ & $E^{\prime \prime}(\mathrm{MPa})$ & $\tan \delta$ & $E_{\mathrm{D}}\left(\mathrm{m} J / \mathrm{mm}^{3}\right)$ \\
\cline { 2 - 5 } & \multicolumn{2}{c}{ Material I } & & \\
\hline 0 (fresh state) & 1.62 & 0.43 & 0.27 & 0.0108 \\
30 & 1.74 & 0.63 & 0.38 & 0.0143 \\
60 & 1.56 & 0.58 & 0.39 & 0.0153 \\
90 & 1.30 & 0.48 & 0.39 & 0.0145 \\
& & & & \\
0 (fresh state) & 11.9 & 3.31 & 0.28 & 0.0175 \\
30 & 13.0 & 4.80 & 0.39 & 0.0185 \\
60 & 10.2 & 3.63 & 0.37 & 0.0174 \\
90 & 9.45 & 3.49 & 0.39 & 0.0187 \\
& & & & \\
0 (fresh state) & 1.64 & 0.94 & 0.57 & 0.0335 \\
30 & 2.19 & 1.31 & 0.60 & 0.0459 \\
60 & 2.78 & 1.62 & 0.58 & 0.0442 \\
90 & 2.52 & 1.44 & 0.57 & 0.0417 \\
\hline
\end{tabular}

\subsubsection{Aging effect}

Fig. 7 and Table 2 present the effect of the aging periods on the mechanical behavior of the three tested materials. Materials I and II have a small variation of all the properties over the aging period, namely concerning the bulk modulus that decreases around $20 \%$ after an aging period of 90 days. Same materials show an increasing of $\tan \delta$ around $40 \%$ after 90 days of aging in synthetic sweat. Material III behaves in a different way, with an increase of the bulk modulus over the aging period, reaching a rise of around $70 \%$ while $\tan \delta$ remains approximately unchanged.

After the process of aging, in general for all three materials, it is noticeable the increase of the energy dissipated per volume unit of material, as well as the increase in the elastic modulus (Table 2).

\subsection{Discussion of the mechanical tests results}

An overall assessment of the materials mechanical characteristics allows concluding that material II has the highest rigidity, especially under dynamic loading, while material III dissipates more energy if compared to the others. These general conclusions are in accordance with the manufacturers recommendations. Actually, interfaces made of material I and material II are recommended to active amputees, with $\mathrm{K}$ levels from $\mathrm{K} 2$ to $\mathrm{K} 4$, which includes amputees from limited mobility (K2) up to amputees with high activity (K4). On the other hand, interfaces made of material III are recommended when an optimal wearing comfort is required, therefore suitable for geriatric, diabetic and sensitive patients. In fact, material III favors the cushion to maximize comfort, despite the dissipated energy may be significant.

Concerning the effect of aging, materials I and II are affected in a similar way, with a significant increase of the rigidity after the 30 days of aging in synthetic sweat followed by a progressive reduction. After 90 days of storage in synthetic sweat, these materials reached a rigidity slightly lower than the initial value. 

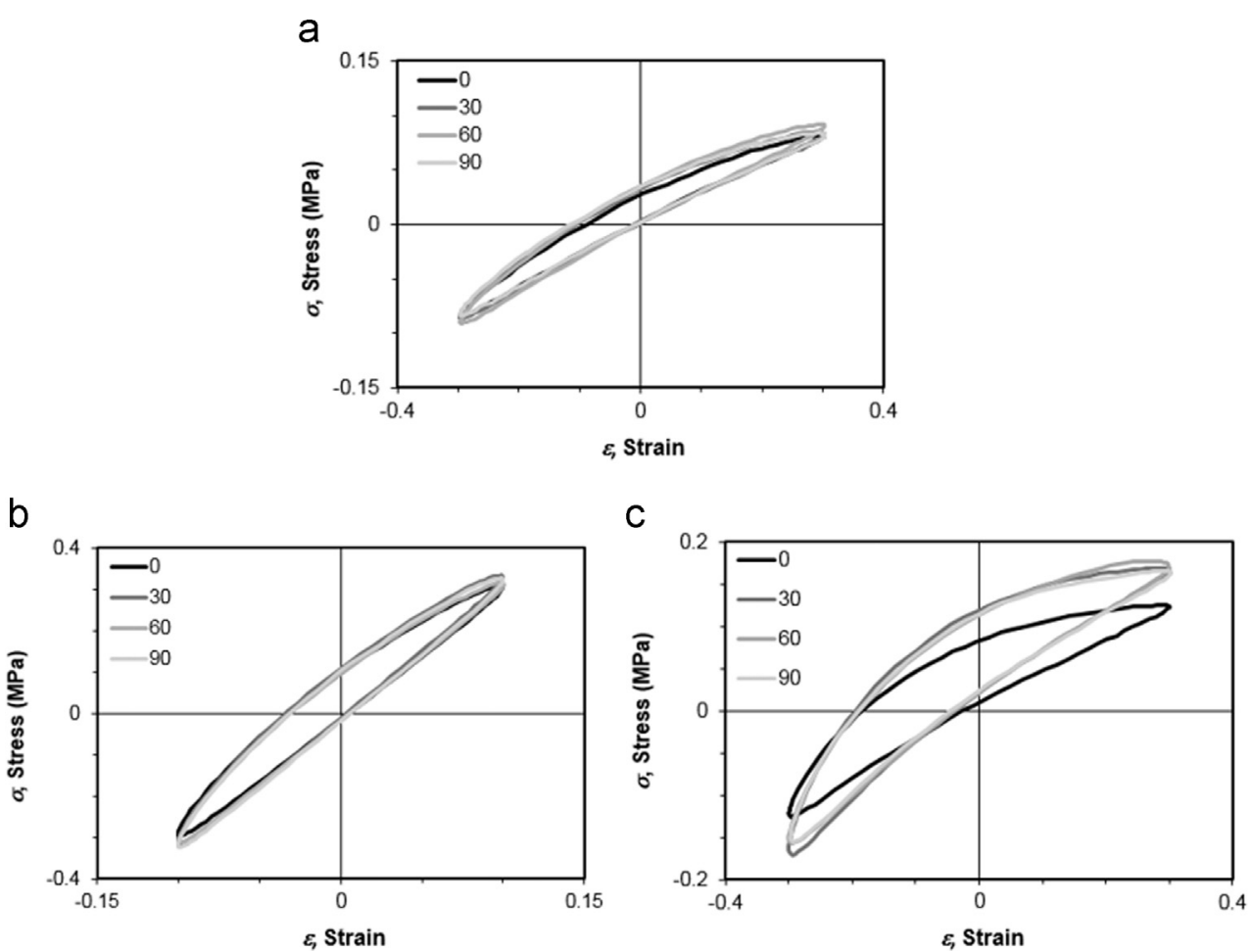

Fig. 7 - Effect of the aging period in synthetic sweat, from 0 to 90 days, on the dynamic mechanical behavior of (a) material I, (b) material II and (c) material III.

Indeed the material III performs in a different way, displaying a continuous increase of rigidity along the 90 days of storage. The variation was similar in both bulk and lost modulus, therefore the $\tan \delta$ values remains almost unchangeable.

The storage in synthetic sweat absolutely induced significant changes in the mechanical behavior of the studied soft interface materials. The more sensitive material was material III, with variations of rigidity around $60 \%$ higher than the original values. Several techniques were used in order to understand the origin of these variations, namely FTIR analysis, $\mathrm{X}$-ray diffraction and morphology characterization by SEM.

\subsection{FTIR analysis}

FTIR was used to assess the chemical structure of the three interface materials studied in this work, in the fresh state and after aged during 90 days in synthetic sweat.

The patent that describes the production of material I (US $6,552,109$ B1) only gives a qualitative general composition of the material. It is a block copolymer with unknown proportions of the blocks, which are styrene and three types of alkenes, ethylene, propylene and butylene. All these monomers are hydrocarbons with $\mathrm{C}-\mathrm{H}$ and $\mathrm{C}-\mathrm{C}$ bonds and, eventually, residual $\mathrm{C}=\mathrm{C}$ bonds; in addition, styrene is an aromatic monomer, thus it has a benzyl group that remains in the structure after the polymerization.

In the spectra of material I, Fig. 8a, the stretching vibration peaks of $\mathrm{C}-\mathrm{H}$ bonds are observed, at several vibration frequencies: at $\sim 2850$ and $\sim 2920 \mathrm{~cm}^{-1}$, from $-\mathrm{CH}_{2}$ groups, and at $\sim 2960 \mathrm{~cm}^{-1}$ from $-\mathrm{CH}_{3}$ groups. On the other hand, the bending vibrations of $\mathrm{C}-\mathrm{H}$ bonds in saturated hydrocarbons are present at $\sim 1370$ and $\sim 1450 \mathrm{~cm}^{-1}$, from $-\mathrm{CH}_{2}$ and $-\mathrm{CH}_{3}$ groups, and between $\sim 700$ and $780 \mathrm{~cm}^{-1}$ from $-\mathrm{CH}_{2}$ groups. Some signs of the presence of residual unsaturated hydrocarbons, due to unreacted alkenes, are seen between 680 and $770 \mathrm{~cm}^{-1}$ and also in the interval $850-1000 \mathrm{~cm}^{-1}$, which can be ascribed to bending vibrations of $\mathrm{C}-\mathrm{H}$ bonds in carbons with double bond ( $\mathrm{C}=\mathrm{CH}_{2}$ groups). All these peaks and bands confirm the presence of saturated hydrocarbons in the sample, as expected, and some unreacted monomers or terminals with $\mathrm{C}=\mathrm{C}$ bonds. In addition, the bending vibrations of the monosubstituted benzene ring of styrene appear as a strong band between 600 and $770 \mathrm{~cm}^{-1}$, which overlaps above $680 \mathrm{~cm}^{-1}$ with the bending vibration bands of $\mathrm{C}-\mathrm{H}$ from $-\mathrm{CH}_{2}$ and $=\mathrm{CH}_{2}$ groups in saturated and unsaturated hydrocarbon chains, as previously discussed. Some perturbation of the base line is also noted near $1490 \mathrm{~cm}^{-1}$, and this match as well with the benzene ring vibration. Other small peaks were found between 1020 and $1150 \mathrm{~cm}^{-1}$ and at $\sim 1250$ and $\sim 1750 \mathrm{~cm}^{-1}$; these may be due to the stretching vibration bands of $\mathrm{C}-\mathrm{O}$ bonds from $-\mathrm{C}-\mathrm{O}$ and $-\mathrm{C}=\mathrm{O}$ groups. The copolymer does not have oxygen in its composition, however the production patent of this material mentions the use of a high amount of a plasticizing oil that can have this type of bonds (esters, carboxylic groups).

Spectra of materials II and III are very similar, Fig. 8b and c, thus they will be described simultaneously. In fact, they are both silicones, corresponding material II to a gel of silicone and material III to a silicone elastomer, as referred in Section 2.1. 
a

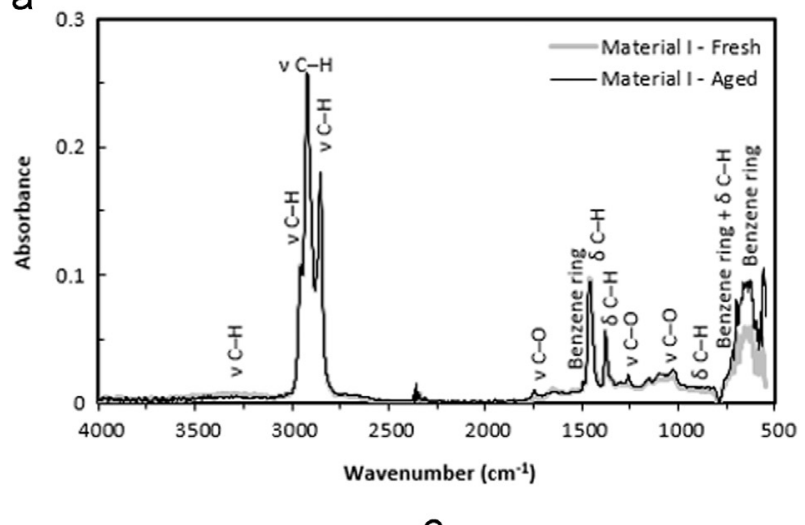

b

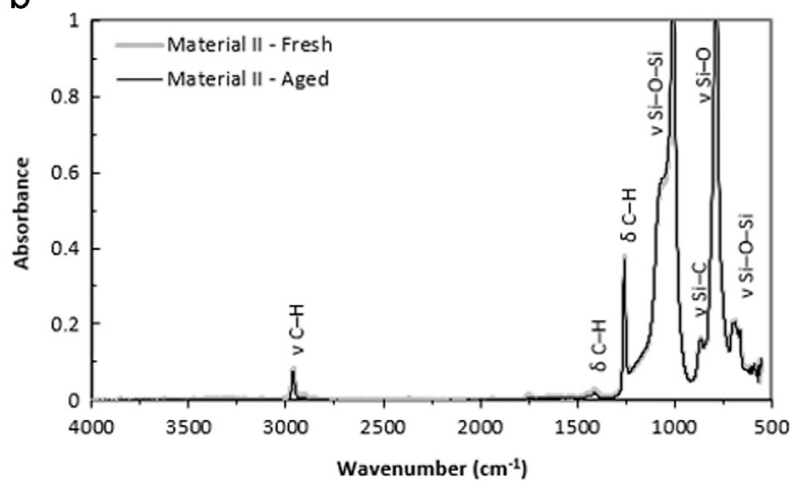

C

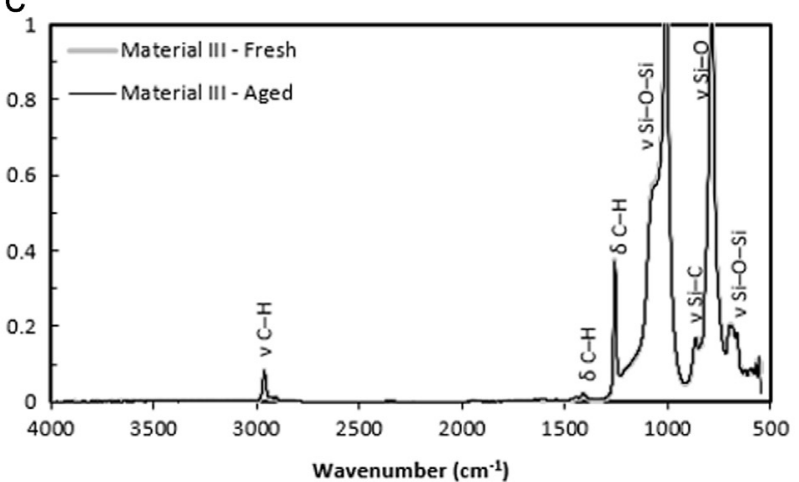

Fig. 8 - FTIR spectra of the interface materials, in fresh state and after being aged: (a) material I, (b) material II and (c) material III.

A stretching vibration band due to $\mathrm{C}-\mathrm{H}$ bonds in $-\mathrm{CH}_{3}$ groups is observed at $\sim 2960 \mathrm{~cm}^{-1}$, indicating the presence of methyl groups in the interface material. These methyl groups are linked to $\mathrm{Si}$, since the bending vibrations of $\mathrm{C}-\mathrm{H}$ in $\mathrm{Si}-\mathrm{R}$ groups appear at $\sim 1410$ and $\sim 1260 \mathrm{~cm}^{-1}$. It is also possible to see the stretching vibration peak of the $\mathrm{Si}-\mathrm{C}$ bonds at $\sim 860$ $\mathrm{cm}^{-1}$. The sharp peak that appears at $\sim 780 \mathrm{~cm}^{-1}$ proves the presence of stretching vibrations of $\mathrm{Si}-\mathrm{O}$ bonds; similarly the peak at $\sim 880 \mathrm{~cm}^{-1}$ and the large peak between 1000 and $1100 \mathrm{~cm}^{-1}$ correspond to the stretching vibrations of the SiO-Si bonds; these peaks can be ascribed to the silica network ( $\equiv \mathrm{Si}-\mathrm{O}-\mathrm{Si} \equiv$ ) in silicones. In summary, the observed chemical groups confirm the existence of a silica-based backbone, with methyl groups linked to Si. This was expected for a silicone structure, $\left[\mathrm{R}_{2} \mathrm{SiO}\right]_{n}$, being $\mathrm{R}$ the methyl groups.

A slight peak or perturbation of the base line, near $2350 \mathrm{~cm}^{-1}$, is observed in the spectrum of some samples, which is due to an interference of $\mathrm{CO}_{2}$ caused by the equipment atmosphere variation during the measuring.

According to the presented results for the three materials, the overlapping of the spectra of fresh and aged samples in all the range is notorious. Thus, it can be concluded that the interface samples did not undergo any significant change of their chemical structure due to the 90 days aging treatment in sweat, proving the good chemical stability of the tested materials in this severe condition. In this way, the materials chemical structure cannot be considered as a preponderant factor for the observed variations in the mechanical behavior of the materials after aging. Further analyses were performed, namely X-ray diffraction (XRD) and scanning electron microscopy (SEM), in order to understand if these variations were based on structural or morphological changes in the materials, as will be presented in the next sections.

\subsection{X-ray diffraction}

As can be observed in Fig. 9 a-c, the XRD patterns obtained for fresh and aged samples of each material are very similar. Materials I and II show a major hump at smaller angles, followed by a very broad and small hump at higher angles. Material III has also a pattern with these two humps, but overlapped with three defined peaks. The humps patterns indicate that all materials have an amorphous structure in their main matrices. However, the additional sharp peaks observed in the XRD pattern of material III demonstrate the presence of crystalline loads. These sharp peaks, when searched in the ICDD database, have matched with synthetic ferrierite (ICDD card 01-082-1396) and coesite (ICDD card 01-076-1805) patterns, both with $\mathrm{SiO}_{2}$ chemical formula. The presence of these crystalline silica phases suggests the addition of fillers or the formation of some phase precipitates during processing.

Neither the humps of materials I $\left(\sim 21^{\circ}(2 \theta)\right)$, II $\left(\sim 14^{\circ}(2 \theta)\right)$ and III $\left(\sim 14^{\circ}(2 \theta)\right)$ nor the narrow peaks of material III $\left(\sim 11^{\circ}\right.$ $(2 \theta)$ and $\left.\sim 34^{\circ}(2 \theta)\right)$ changed their location with the aging 

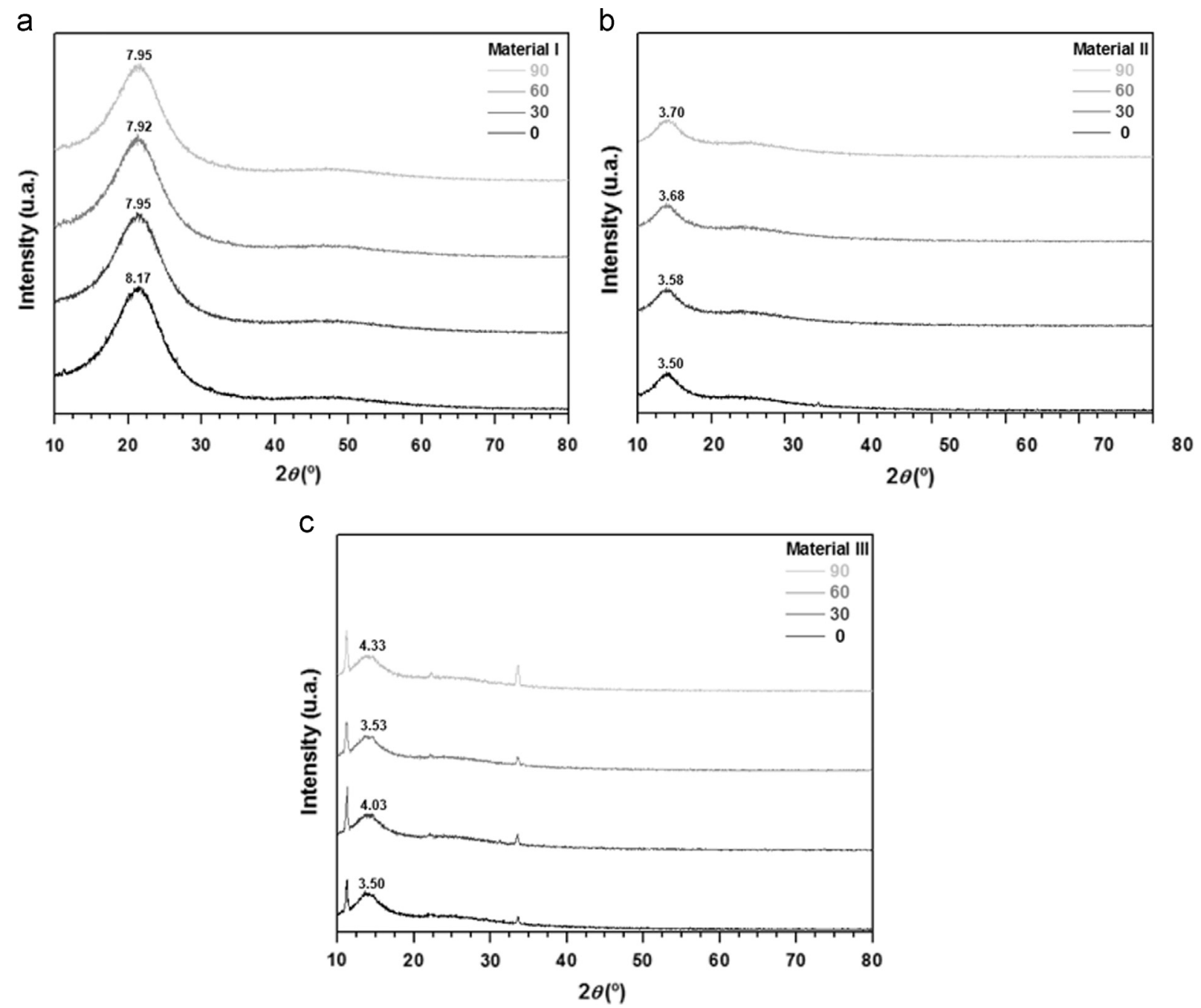

Fig. 9 - XRD patterns and FWHM values for the interface materials, aged from 0 to 90 days in synthetic sweat: (a) material I, (b) material II and (c) material III.

process. This indicates that the main phases composition has not changed due to aging. This is in agreement with the FTIR results.

Thus, for all the samples the Full Width at Half Maximum (FWHM) of the major humps was evaluated, as a measurement of the structural organization. Widest FWHM humps indicate a more amorphous phase. Contrary, a higher crystallinity level of the main phase can be inferred by the humps narrowing.

With aging, the FWHM of material I patterns suffers a slight reduction, already in the first 30 days of aging, which demonstrates an increase of crystalline order in the polymer chains that may induce a slight increase in the rigidity of the polymer. The FWHM of material II patterns suffers a small rise that suggests an increase of amorphization state in the silica network. However, both of the FWHM variations do not show significant values to affirm that the materials structure suffers modification with aging. They just show a tendency that cannot fully justify the mechanical behavior results, if compared with the elastic modulus variation magnitudes.
In material III, the XRD patterns show a hump's FWHM bigger, unlike the peaks' FWHM that remains stable. This increase reveals, as in material II (of the same type), an amorphization of the silica network, The hump's FWHM variation confirms the sensibility of this material to the chemical aging when in contact with human sweat. This higher sensibility is also noticed in the mechanical behavior changes.

In summary, there are observable changes in the degree of organization of the materials matrices upon aging. These variations may be in some extent related to the changes in the mechanical behavior of the materials. It is worth noting that the two silicone materials tend to get more amorphous with aging, and this may be induced by the introduction of some defects in the silica network (siloxane bonds rupture), which can be due to the incorporation of large ions (e.g. $\left.\mathrm{Na}^{+}\right)$ of the sweat solution in the network.

\subsection{Morphological analysis by SEM}

From SEM observation (Fig. 10), it was possible to verify that over 90 days of aging no significant changes in the samples 
a1
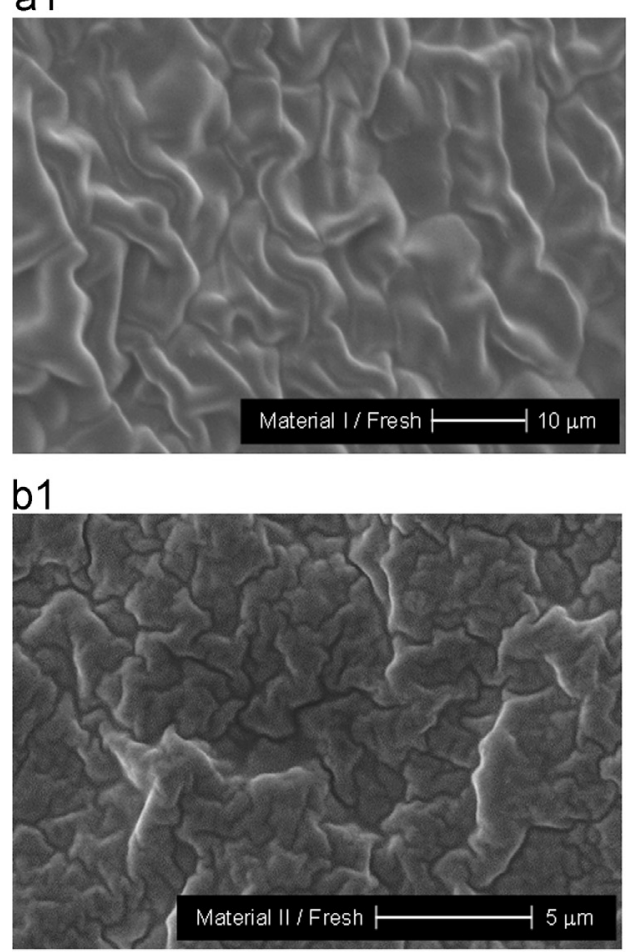

c1

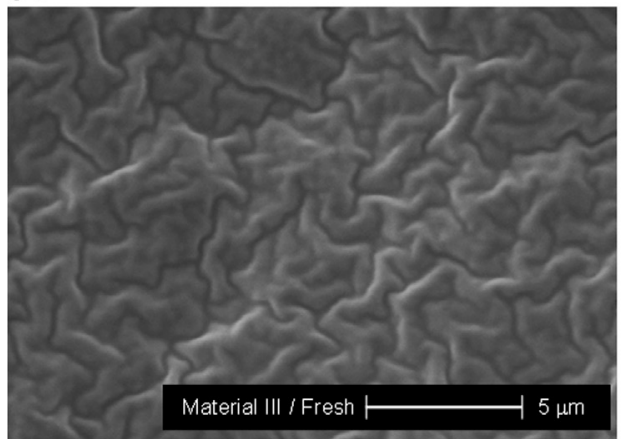

a2

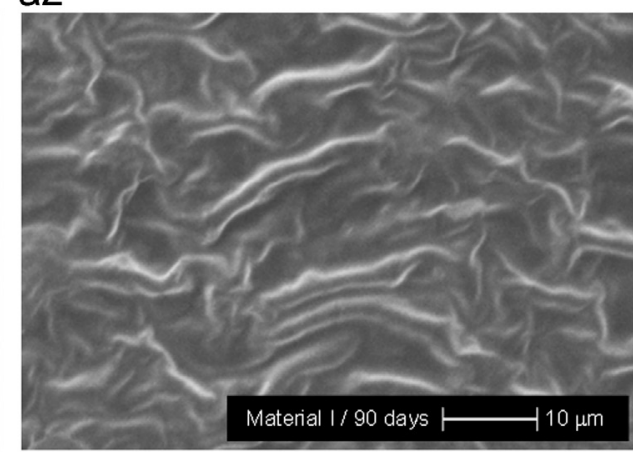

b2

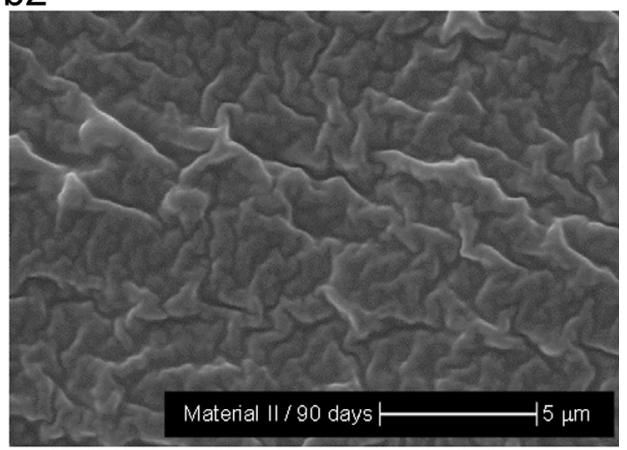

c2

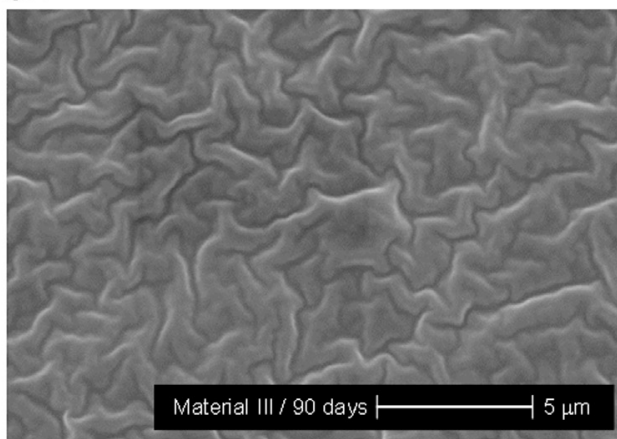

Fig. 10 - Surface morphology of materials I, II and III, in fresh condition and after 90 days of storage in synthetic sweat.

surface were found. This reveals that the morphology of the samples was not altered with aging.

\section{Conclusions}

The mechanical behavior of three commercial interface materials for transtibial prosthesis was investigated in fresh condition and after aging periods up to 90 days on a synthetic sweat. The chemical composition, the structure and the morphology were also evaluated in order to help explain the variation of interface's mechanical behavior along the aging period. It was concluded that the three tested materials behave with a linear response for small deformations followed by a nonlinear behavior for higher deformation. Moreover, all the three materials have strong viscoelastic behavior, displaying an increase of Young's modulus with the loading rate/regime.
Particularly, material II has the highest rigidity, especially under dynamic loading, while material III dissipates more energy if compared to the others. These properties are in accordance with the manufacturers' recommendations, i.e. interfaces made of materials I and II are recommended to active amputees (K3-K4), while interfaces made of material III are recommended when an optimal wearing comfort is required (K1-K2).

Concerning the aging effect, materials I and II are affected by aging in synthetic sweat in a similar way, with a significant increase of their rigidity after the 30 days, followed by a progressive reduction, reaching rigidity slightly lower than the initial value after 90 days. Material III displays a different behavior, with a continuous increase of rigidity along the 90 days of storage.

It was also concluded that any of the three interface materials shows significant change of their chemical structure associated with the aging treatment in synthetic sweat, demonstrating their chemical stability under service 
conditions. Similarly, no influence of the aging was noticed on materials morphology.

Discreet variations on XRD patterns of the materials I and II reveal that their structure organization only suffers modest alterations with aging. However, material III shows a bigger hump's FWHM variation that indicates a higher sensibility of this material to aging.

Indeed the material that showed greater sensitivity to the effect of aging on their properties was material III. This material presents the lowest stiffness, being suitable for uses requiring maximum comfort. Our results seem to indicate that aging affects in larger extent the materials that initially have lower stiffness.

\section{Acknowledgments}

The authors thank the company IDEO SL (Seville - Spain) for providing the analyzed samples.

\section{R E F E R E N C E S}

Al-Oweini, R., El-Rassy, H., 2009. Synthesis and characterization by FTIR spectroscopy of silica aerogels prepared using several $\mathrm{Si}(\mathrm{OR})_{4}$ and R"Si(OR') ${ }_{3}$ precursors. J. Mol. Struct. 919, 140-145.

Becker, H.G.O., Berger, W., Domschke, G., Fanghänel, E., Faust, J., Fischer, M., Gentz, F., Gewald, K., Gluch, R., Mayer, R., Müller, K., Pavel, D., Schmidt, H., Schollberg, K., Schwetlick, K., Seiler, E., Zeppenfeld, G., 1997. Organikum, second ed. Calouste Gulbenkian Foundation, Lisbon.

Berge, J., Czerniecki, J., Klute, G., 2005. Efficacy of shock-absorbing versus rigid pylons for impact reductionin transtibial amputees based on laboratory, field, and outcome metrics. JRRD 42 (6), 795-808.

Berke, H.G., 2007. Transtibial Prostheses. In: Lusardi, M., Nielsen, C. (Eds.), Orthotics and Prosthetics in Rehabilitation. Saunders Elsevier, Filadelfia, pp. 679-710.

Carvalho, J.A., 2003. Amputações De Membros Inferiores - Em Busca Da Plena Reabilitação, second ed. Manole, São Paulo.

Covey, S., Muonio, J., Street, G., 2000. Flow constraint and loading rate effects on prosthetic liner material and human tissue mechanical response. JPO 12 (1), 15-32.

De Godoy, J.M.P., Braille, D.M., Buzatto, S.H.G., Longo, O., Fontes, O.A., 2004. Quality of life after amputation. Psychol. Health Med 7 (4), 397-400.

Emrich, R., Slater, K., 1998. Comparative analysis of below-knee prosthetic socket liner materials. J. Med. Eng. Technol. 22 (2), 94-98.

Huang, M., Levy, C., Webster, J., 2001. Acquired limb deficiencies: 3. Prosthetics components, prescriptions, and indications. Arch. Phys. Med. Rehabil. 82 (1), 17-24.
Klute, G., Kallfelz, C., Czerniecki, J., 2001. Mechanical properties of prosthetic limbs: adapting to the patient. JRRD 38 (3), 299-307.

Leite, V.M.F., Pisani, M.X., Paranhos, H.F.O., Souza, R.F., SilvaLovato, C.H., 2010. Effect of ageing and immersion in different beverages on properties of denture lining materials. J. Appl. Oral. Sci 18 (4), 372-378.

Levy, S., 1980. Skin problems of the leg amputee. Prosthet. Orthot. Int. 4, 37-44.

Linde, H., Hofstad, C., Geurts, A., Postema, K., Geertzen, J., Limbeek, J., 2004. A systematic literature review of the effect of different prosthetic components on human functioning with a lower-limb prosthesis. JRRD 41 (4), 555-570.

Lunsford and Contoyannis, 2009. Ciencia de los materiales in Hsu, Michael \& Fisk. AAOS Atlas de Ortotesis y Dispositivos de Ayuda. Elsevier España, S.L, Barcelona15-51.

Mak, A., Zhang, M. e, Boone, D., 2001. State-of-art research in lower-limb prosthetic biomechanics-socket interface: a review. JRRD 38 (2), 161-174.

Martín, M., Pérez, F., 2009. Epidemiologia de la amputación. In: Periago, R. (Ed.), Prótesis, Ortesis Y Ayudas Técnicas. Elsevier España, SL, Barcelona, pp. 15-19.

Meulenbelt, H., Geertzen, J., Jonkman, M., Dijkstra, P., 2009. Determinants of skin problems of the stump in lower-limb amputees. Arch. Phys. Med. Rehabil. 90 (1), 74-81.

Pitkin, M., 2010. Biomechanics of Lower Limb Prosthetics. Springer, Berlin.

Ramos, A., Mendonça, A., Okamoto, A., Ingham, S., 2007. Amputações. In: Fernandes, A., Ramos, A., Casalis, M., Herbert, S. (Eds.), AACD - Medicina e reabilitação: princípiosEe Prática. Artes Médicas, São Paulo, pp. 207-229.

Sanders, J., Greve, J., Mitchell, S., Zachariah, S., 1998. Material properties of commonly-used interface materials and their static coefficients of friction with skin and socks. JRRD 35 (2), 161-176.

Sanders, J., Zachariah, S., Baker, A., Greve, J., Clinton, C., 2000. Effects of changes in cadence, prosthetic componentry, and time on interface pressures and shear stresses of three transtibial amputees. Clin. Biomech. 15, 684-694.

Sanders, J., Nicholson, B., Zachariah, S., Cassisi, D., Karchin, A., Fergason, J., 2004. Testing of elastomeric liners used in limb prosthetics: classification of 15 products by mechanical performance. JRRD 41 (2), 175-186.

Selinger, D., 2004. Weight of prefabricated transtibial gel prosthetic liners - a standardized sample. Resident Research Project. California State University, 1-17.

Sinha, R., Van den Heuvel, W., Arokiasamy, P., 2011. Factors affecting quality of life in lower limb amputees. Prosthet. Orthot. Int. 35, 90-96.

van de Weg, F., van de Windt, D., 2005. A questionnaire survey of the effect of different interface types on patient satisfaction and perceived problems among trans-tibial amputees. Prosthet. Orthot. Int. 29 (3), 231-239. 